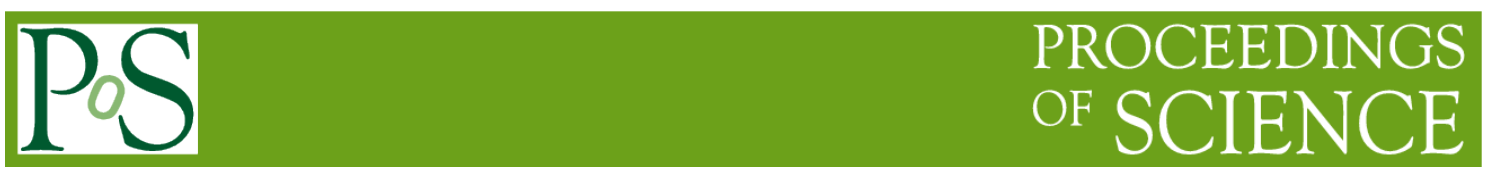

\title{
HD2UB: A Voice Communication System for Underground Mine Monitoring
}

\author{
Yupeng Yang ${ }^{1}$ \\ College of Computer Science and Technology on Computer Vision and Image Processing Laboratory \\ Jilin University, Changchun, 130012, China \\ E-mail:yangyp920@gmail.com
}

\section{Wenhui Li, Huiying $\mathbf{L i}^{2}$, Jinlong Zhu}

College of Computer Science and Technology on Computer Vision and Image Processing Laboratory Jilin University, Changchun, 130012, China

E-mail: lihuiyingejlu.edu.cn

The voice communication is an important application in WSNs. The weakness and limitations of the communication distance of traditional systems are the main challenge, especially in complex underground (UG) environment. We provide a voice communication system named HD2UB, which adopts CML's CMX618 for voice data collection and forward error correction coding, TI's CC2530 for ZigBee network formation and voice data transmission. We assess the system from the perspective of Point to Point (P2P), End to End(E2E) and energy. The result shows that the HD2UB ensures voice quality in complex ZigBee network with higher integration and lower power consumption when compared with the existed ZigBee voice system.

CENet2015

12-13 September 2015

Shanghai, China

\footnotetext{
${ }^{1}$ Speaker

${ }^{2}$ Corresponding Author
} 


\section{Introduction}

Wireless sensor networks (WSNs) are the hot research field which integrate micro-sensors with monitor real-time collaboration, perception and collection of a variety of environmental or monitoring information. These terminals transmit information by multi-hop wireless network $[1,2]$. Sensor networks are mainly used in the environmental monitoring, industry, bio-medical and other fields [3]. In the mine safety monitoring, for example, UG collapse can cause structural variations which can be detected by a WSN system named SASA. Mo Li et al developed a reliable and stable mechanism to find out the relocated sensor nodes in the singlehole region and to handle queries under unstable circumstances [4]. In addition, the need of emergency rescue cannot be satisfied with the traditional wired data transmission, thus the sensor nodes with the voice communication capabilities should also be able to ensure the voice communication quality between the base stations and undermine the personal handheld nodes [5] and video, etc. to monitor UG environment and the safety of persons undermine [6]. The UG environment limits the transmission range and makes it different from in terrestrial because of water content, soil composition, density and burial depth of sensor devices, etc. [7]. UG networks cannot transmit straight. If some nodes cannot work, the others will link the network. In summary, this paper makes contribution from the following aspects: 1) a new kind of voice communication system (HD2UB, a handheld voice node to above-ground (AG) base station) is presented, which makes smooth communication in UG coal mine via multi-hop ZigBee networks; 2) this paper introduces the hardware design which consists of handheld voice nodes, router nodes, and gateway in mesh ZigBee networks; 3) this paper puts forward a communication mechanism. Besides, lots of experiments and tests have been carried out to improve the voice quality of the communication mechanism.

\section{Related Works}

In the design of voice node, Crossbow developed TMTS300CA and MTS310CA, such two sensor nodes with integrated light, temperature, voice, buzzer and magnetometer acquisition node in order to connect Mica, MicaZ sensor nodes series [8]. With an adaptive noise cancellation method, Cao et al proposed a voice communication system which reduces noise in communication, including the FPGA chip for PCM voice collection and the TMS320 VC5402 components for speech enhancement [9]. The voice nodes were designed by using LPC2138 with ARM microcontrollers and CMX618 [10]; The base station with CMX7041 and voice nodes with CMX618 was proposed to accord with DPMR radio standards [11].

The existing designs of voice nodes consider less on the complexity of the speaking environment, especially in UG mines [12]. Most of the voice communication is based on IEEE802.15.4. To a large extent, voice systems are proposed in a star ZigBee network, just for point to point communication (P2P), not to mention the need of the multi-hop voice communication (especially in mesh networks $[13,14]$ ), and the coverage of voice signal is so limited. To overcome the above limitation, this paper uses CMX618 as the voice-core chip and CC2530 as the wireless transmission chip.

\section{Hardware for HD2UB}

The design of the HD2UB system hardware consists of three important parts: the handheld voice node, the router node and the gateway.

The handheld voice node contains three parts: audio module, wireless transmission module and power module. Audio module includes CMX618 chip and microphone and speaker; wireless communications module using TI's CC2530; power module, use 3 AA batteries. CMX618 can use RALCWI (Robust Advanced Low Complexity Waveform Insert) voice compression algorithm [9], which provides very low rate of toll-quality performance. CC2530 is a true system-on-chip, including the RF transceiver, industry-standard enhanced $8051 \mathrm{MCU}$, the system programmable flash memory, 8KB RAM and many other features. CMX618 and 
$\mathrm{CC} 2530$ are connected via C-BUS, which is a four-line interrupt-driven serial bus system. The control and status information is sent by main controller through the C-Bud; besides, in order to meet the actual application requirements, the handheld node connects to the power button, intercom button (divided into idle, sending, receiving three status), LED indicator (display the node status), EEPROM memory module (if it is unnecessary of demanding a lot of voice data buffer, the CC2530 flash memory is quite enough) and an antenna. In order to allow users enjoying better voice quality, voice node links the TDA2822 amplifier circuit between the CMX618 and speaker's connection. Fig.1 shows the modules.

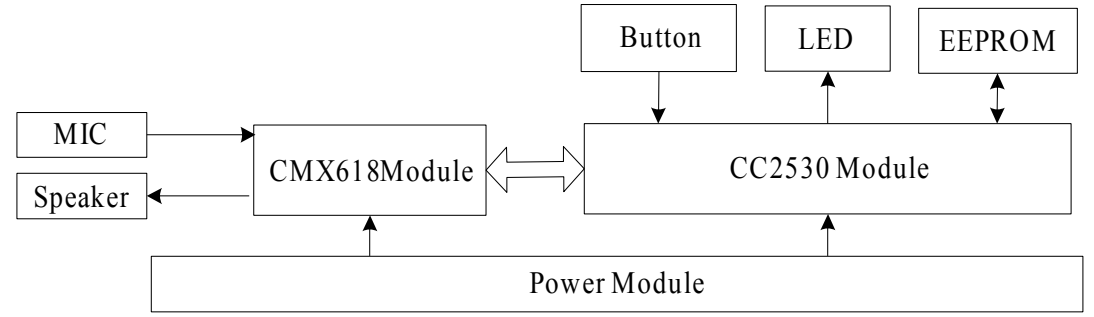

Figure 1: Modules of Voice Node

Router nodes transmit the voice packet with the following ZigBee protocol. The router node is designed only with the CC2530 chip and the associated circuitry, the antenna and the battery. In order to prevent environmental damages to the router node, we add a protective cover outside to protect the router nodes. A gateway is needed in the AG monitor center. We also design a gateway to build the ZigBee network as a coordinator and gather the voice sent from UG. All the voice should be sent to the gateway. The user in the AG base station with the handheld voice node can get the voice from the gateway. Use LM3S9B96 and CC2520 to develop the gateway. The related work about the gateway design will be specifically proposed in another article.

\section{Communication Mechanism}

The voice communication is mainly between the base station and the handheld mobile nodes. Fig. 2 and Fig. 3 show the communication mechanism

Voice intercom is divided into three phases: the request phase, the intercom phase and the idle phase.

In the request phase, it automatically sends a voice request message to the base station after turning on the power of the handheld voice node. The base station receives message through the multi-hop ZigBee network and replies the confirmed message to the handheld node.

In the Intercom phase, CMX618 of the mobile node is in encoding mode, and every $80 \mathrm{~ms}$ generates $288 \mathrm{~b}(36 \mathrm{~B})$ voice data, which produces a Voice Access Interrupt (VAI). VAI notifies CC2530 MCU to store the collected voice data in the transmit queue buffer. After 5 VAI, a Data Transmission Interrupt (DTI) is generated which announces that the MCU in CC2530 get the voice data $(36 \mathrm{~B} * 5)$ encapsulated to meet the ZigBee standards frame format. The data packets are sent to ZigBee networks through the RF in CC2530. 


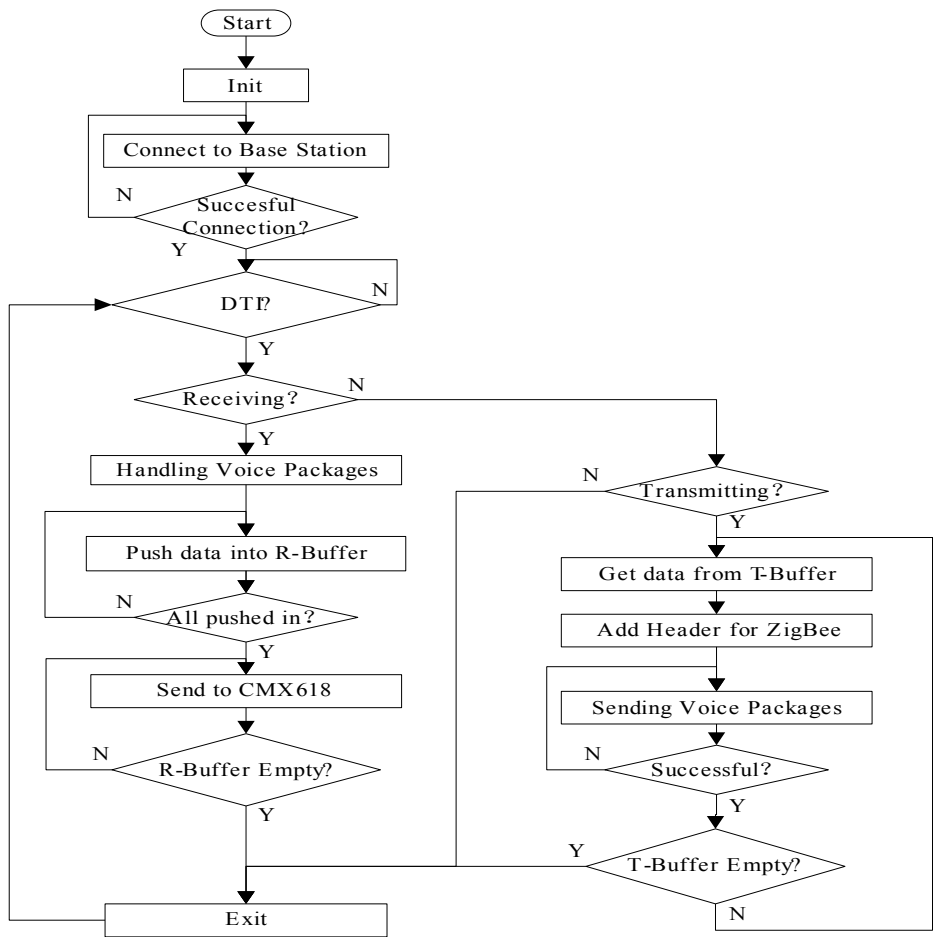

Figure 2: Process of Coding and Decoding

In the idle phase, both CMX618 and CC2530 are in the Idle Status. The handheld node cannot transmit or receive the voice data.

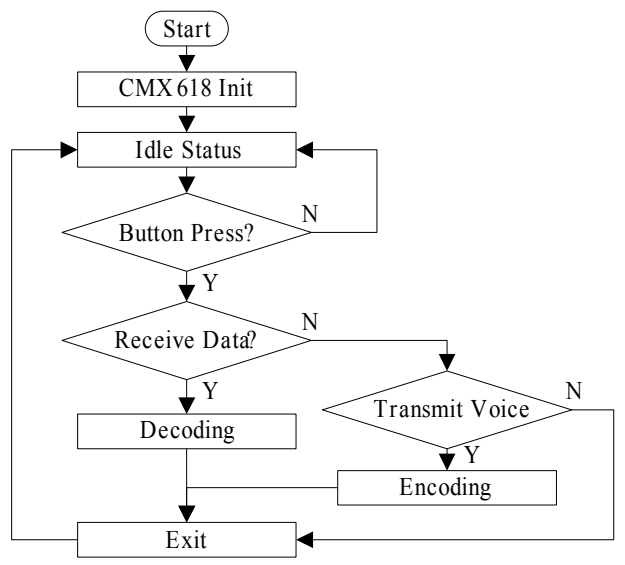

Figure 3: Communication Mechanism

\section{Tests and Experiments}

Choose TI SmartRF Sniffer Software to detect voice data in ZigBee networks. According to the ITU-T G.107 standard [15], we assess the voice node from Point to Point communication ability, End to End communication ability and Energy Analysis.

\subsection{P2P Communication Tests}

Test is carried out in the open ground without wind and at the temperature of $0^{\circ} \mathrm{C}$. We set up one voice node to keep the transmission of 1000 data packets to another voice node. We test the communication per 5 meters from 0 to 50 meters. Record the loss rate(LR) of packets in Table 1. 


$\begin{array}{llllllllll}\mathrm{D}[\mathrm{m}] & \mathrm{LR}[\%] & \mathrm{D}[\mathrm{m}] & \mathrm{LR}[\%] & \mathrm{D}[\mathrm{m}] & \mathrm{LR}[\%] & \mathrm{D}[\mathrm{m}] & \mathrm{LR}[\%] & \mathrm{D}[\mathrm{m}] & \mathrm{LR}[\%] \\ 5 & 0.0 & 20 & 1.1 & 35 & 3.9 & 38 & 5.4 & 45 & 8.9 \\ 10 & 0.1 & 25 & 2.5 & 36 & 4.5 & 39 & 6.0 & 50 & >10 \\ 15 & 0.6 & 30 & 3.2 & 37 & 4.9 & 40 & 6.5 & \text { D:Distance }\end{array}$

Table 1: Loss Rate of HD2UB

$\begin{array}{llllllllll}\mathrm{D}[\mathrm{m}] & \mathrm{LR}[\%] & \mathrm{D}[\mathrm{m}] & \mathrm{LR}[\%] & \mathrm{D}[\mathrm{m}] & \mathrm{LR}[\%] & \mathrm{D}[\mathrm{m}] & \mathrm{LR}[\%] & \mathrm{D}[\mathrm{m}] & \mathrm{LR}[\%] \\ 5 & 0 & 20 & 0 & 35 & 0 & 38 & 3 & 41 & 100 \% \\ 10 & 0 & 25 & 0 & 36 & 0 & 39 & 21 & & \\ 15 & 0 & 30 & 0 & 37 & 0 & 40 & 100 & \text { LR: Loss Rate }\end{array}$

Table 2: Loss Rate of Wireless Voice Communication System

Table 1 shows that the loss rate in $30-35 \mathrm{~m}$ is tolerable. In the design, mass of router nodes is deployed in the environment. The distance between two router nodes is less than $10 \mathrm{~m}$. The system also adopts CC2530 as the main chip [16]. Its loss rate keeps zero within $37 \mathrm{~m}$ communication distance, HD2UB's loss rate grows very slowly with distance growing. In $37 \mathrm{~m}$, $38 \mathrm{~m}$ and $39 \mathrm{~m}$, our LR is $5.4 \%, 6.0 \%$ and $6.5 \%$ respectively. From $38 \mathrm{~m}$ to $39 \mathrm{~m}$, its LR grows from $3 \%$ to $21 \%$ violently. Even it fully lose the package in $40 \mathrm{~m}$, whereas HD2UB's LR is $6.5 \%$. Though HD2UB has a little loss rate within $37 \mathrm{~m}$. With the distance growing, it can meet the requirements and perform more stably than the system [16].

The design of buffer length and coding rate is also the key point to voice node performance, so in this section, we have carried out an experiment under the same environment as above to decide the buffer length and coding rate. We use the voice node to transmit $1 \mathrm{~min}$ voice data, with changing length of buffer and coding rate.

\begin{tabular}{|c|c|c|c|}
\hline \multirow{2}{*}{ Buffer } & \multirow{2}{*}{$\begin{array}{l}\text { Coding } \\
2050 \mathrm{~b} / \mathrm{s}\end{array}$} & \multicolumn{2}{|c|}{ Rate } \\
\hline & & $2400 \mathrm{~b} / \mathrm{s}$ & $2750 \mathrm{~b} / \mathrm{s}$ \\
\hline 1000B & $148 \mathrm{~ms}$ & $282 \mathrm{~ms}$ & $403 \mathrm{~ms}$ \\
\hline 2000B & $232 \mathrm{~ms}$ & $364 \mathrm{~ms}$ & $693 \mathrm{~ms}$ \\
\hline $3000 \mathrm{~B}$ & $422 \mathrm{~ms}$ & $948 \mathrm{~ms}$ & $>1000 \mathrm{~ms}$ \\
\hline
\end{tabular}

Table 3: Performance under Different Buffer Lengths and Coding Rates

With a certain buffer size, if the voice coding rate is higher, the voice quality will be better and the delay will be greater; with a certain coding rate, if the buffer is larger, the voice quality becomes poorer and the delay will be more significant. 2000B buffer length and 2400b/s coding rate are chosen to meet the real-time and voice quality .

\subsection{E2E Communication Tests}

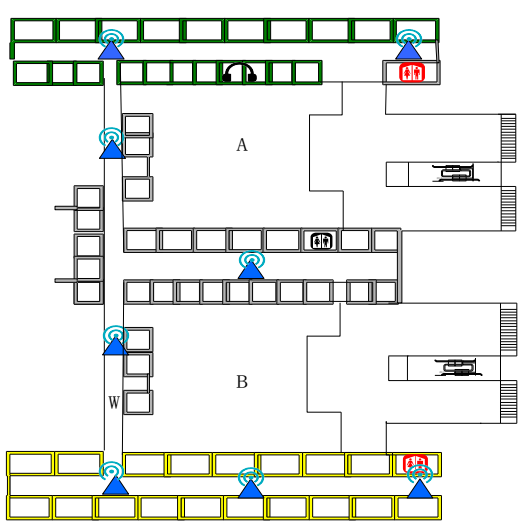

(a)

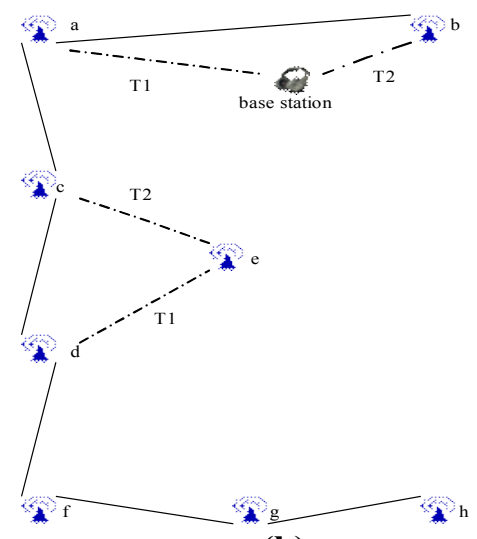

(b)

Figure 4: (a) the real picture of voice node, router node and gateway (b) the topologies 
Test environment of E2E communication ability simulates UG mine in Fig4. Choose a house layer to build a ZigBee network. The network consists of one voice base station and eight router nodes. Deploy the nodes on the ceiling, in the corner or somewhere else to simulate the complexity of the mine environment. Two routers' distance is $10 \mathrm{~m}$ or more. In the test, one person (playing the miner's role) takes the voice node and walks along the corridor while talking to the base station (playing the remote monitoring center) through ZigBee network. The data packet is added with a timestamp to calculate the delay. The hops and the arrival time of the packet are recorded by the base station. The topology may change because of the terrible environment of UG mine, so two topologies $(\mathrm{T} 1, \mathrm{~T} 2)$ are tested in Fig. 4 (b). Table 4 records the latency in different topologies and different hops. The result indicates that the changeable topology barely affects the latency and the voice quality. In the mine, we deploy redundant nodes to make the ZigBee network communication robust. The topology cannot change much. With better performance of the latency, the whole voice can be heard through the HD2UB; in this sense, it can be applied at real mine.

\begin{tabular}{lllll}
\hline Hops & Latency & Voice quality (TI) & Latency & Voice quality (T2) \\
\hline 0 & $269 \mathrm{~ms}$ & Clear & $304 \mathrm{~ms}$ & Clear \\
1 & $590 \mathrm{~ms}$ & Clear & $485 \mathrm{~ms}$ & Clear \\
2 & $1.625 \mathrm{~s}$ & Clear & $1.681 \mathrm{~s}$ & Clear \\
3 & $1.957 \mathrm{~s}$ & Little noisy & $2.148 \mathrm{~s}$ & Little noisy \\
4 & $2.423 \mathrm{~s}$ & Little noisy & $2.359 \mathrm{~s}$ & Little noisy \\
5 & $4.217 \mathrm{~s}$ & Large, complete & $4.531 \mathrm{~s}$ & Large, Complete \\
\hline
\end{tabular}

Table 4: Latency and Voice Quality in T1 and T2 Respectively

\subsection{Energy Analysis}

\begin{tabular}{lll} 
Capacity & Voice-Talk time & Router time \\
$1000 \mathrm{mAh}$ & 16 hours & 0.75 years \\
$1500 \mathrm{mAh}$ & 24 hours & 1.125 years \\
$2000 \mathrm{mAh}$ & 32 hours & 1.50 years \\
$2500 \mathrm{mAh}$ & 40 hours & 1.875 years \\
\hline
\end{tabular}

Table 5: Voice-talk Time and Router Time with Different Battery Capacities

Table 5 shows the predicted lifetime based on measured current drawn from the handheld voice node and the router node board. The board typically draws on average $3.3 \mathrm{~mA}$ at 3 volts while idle and $27 \mathrm{~mA}$ while actively. Using different AA batteries in the calculation, the voice lifetime of voice nodes reaches the requirement of 24 hours. The router node can last for 1-2 years in the power saving mode. If we choose two D-cell batteries at 4-5 years, it can easily meet our requirement (double just for safe) of a 1-2 years standby time of router nodes and over 48 hours talk time for voice node.

\section{Conclusion}

This paper presents HD2UB, a more suitable UG-AG voice communication system in coal mine with ZigBee networks. This system stresses the voice quality in mesh ZigBee with multiple hops for mine monitoring. In addition, the proposed system provides the hardware design of handheld voice node and the communication mechanism. The availability and efficiency of the system has been proved through lots of experiments. These experiments show that HD2UB performs more stably, ensure the voice quality clear and lower energy consumption. Further studies should be carried out to better represent and develop more functions of the system's gateway to achieve the voice, video and other communications for the mine monitoring.

\section{References}


[1] J. Yick, B. Mukherjee, and D. Ghosal. Wireless sensor network survey[J].Computer Networks.52(12),2292-2330(2008).

[2] L. Herrera, A. Calveras, and M. Catalan. A two-way radio communication across a multi-hop wireless sensor network based on a commercial IEEE 802.15.4 compliant platform [J], Procedia Engineering, 25(1): 1045-1048( 2011)

[3] D.De, M.Das Gupta, and A.Sen.Energy efficient target tracking mechanism using rotational camera sensor in WMSN[J]. Procedia Technology.1(4), 674-681(2012)

[4] M. Li, and Y. Liu. Underground Coal Mine Monitoring with Wireless Sensor Networks[J], ACM Transactions on Sensor Networks (TOSN).5(2): 777-781 (2009).

[5] B. S. Nie,W. X. Chen, L. K.Wang, R. M. Zhang, and C. Wang, Internet of Things-Based Positioning of Coalmine Personnel and Monitoring of Emergency State[C],Digital Manufacturing and Automation (ICDMA),2011 Second International Conference on.IEEE, 657-660(2011).

[6] D. Wen, X.Yue,H. F. Ma and Y. T. Wang,Design of Coal Mine Gas Monitoring System Based on ZigBee[C], 2011 International Conference on Future Computer Science and Education,IEEE, USA.pp,580-583 (2011).

[7] Z. Sun and I. F. Akyildiz, Connectivity in Wireless Underground Sensor Networks[C].2010 7th Annual IEEE Communications Society Conference on Sensor Mesh and Ad Hoc Communications and Networks. IEEE, 1-9(2010).

[8] E. Touloupis, A. Meliones, S. Apostolacos. Speech codecs for high-quality voice over ZigBee applications: Evaluation and implementation challenges[J], Communications Magazine, IEEE, 50(4), 122-128(2012).

[9] X. L. Cao, P. Wu and T. F. Ding, Design of a Speech Processing System Based on DSP[J]. Journal of Scientific Instrument, 26,83-584(2005) (In Chinese).

[10]Z. Chai, X. M. Xie, Digital Voice Communication System Based on CMX618[J].Modern Electronics Technique,7,99-110(2009) (In Chinese).

[11] Z. Cui and X. Xie, The design of baseband system for digital interphone based on CMX618 and CMX7041[J]. Application of Integrated Circuits, 35(5),69-73(2009) (In Chinese).

[12] H. Y. Song and S. H. Cho, Performances of IEEE 802.15.4 unslotted CSMA-CA for voice communications [C].17th Asia-Pacific Conference on Communications (APCC).IEEE. Malaysia.pp,151-156(2011).

[13] T. M. Trung and J. Mo. A new scheme to support voice call over wireless multi-channel MAC protocol $[\mathrm{C}]$. First International Conference on Communications and Electronics, IEEE, Vietnam.pp,53-57(2006).

[14] T. M.Trung and J. Mo. VMcMAC:Voice call support over wireless multi-channel MAC protocol[J]. Communications Letters, IEEE 11(5),402-404 (2007)

[15] H. Huang, Y. Wang, Study of Objective Quality Evaluation for the Speech Systems[J].Acta Electronica Sinica, 28(4):112-114(2000).

[16] D. H. Wang, Q. Q. Zhang and Y. F. Sun. Design of Wireless Voice Communication System in Underground Coal Mine Based on ZigBee[C]. Applied Mechanics and Materials. Trans Tech Publications, Switzerland. pp, 548-549,1402-1406(2014). 